\title{
Grey double exponential smoothing model and its application on pig price forecasting in China
}

\author{
Lifeng $\mathrm{Wu}^{* a, b}$, Sifeng Liu ${ }^{a, c}$, Yingjie Yang ${ }^{c}$ \\ ${ }^{a}$ College of Economics and Management, Nanjing University of Aeronautics and Astronautics, \\ Nanjing 210016, China \\ ${ }^{b}$ School of Economics and Management, Hebei University of Engineering, Handan 056038, China \\ ${ }^{c}$ Centre for Computational Intelligence, De Montfort University, Leicester, LE1 9BH, UK
}

\begin{abstract}
:
To resolve the conflict between our desire for a good smoothing effect and desire to give additional weight to the recent change, a grey accumulating generation operator that can smooth the random interference of data is introduced into the double exponential smoothing method. The results of practical numerical examples have demonstrated that the proposed grey double exponential smoothing method outperforms the traditional double exponential smoothing method in forecasting problems.
\end{abstract}

Keywords: forecasting; double exponential smoothing model; accumulating generation operator

\section{Introduction}

The exponential smoothing (ES) method describes a class of forecasting methods. Each has the property that forecasts are weighted combinations of past observations, where recent observations are given relatively more weight than older ones. The double exponential smoothing (DES) is an extension of ES designed for trend time series [1]. The Holt-Winters method (triple exponential smoothing) takes into account both seasonal changes and trends. They provide good forecasts with simple formulations, allowing the incorporation of error, trend and seasonal components in

${ }^{0 *}$ Corresponding Author: E-mail address: wlf6666@126.com. 
a comprehensive manner [2-5]. Their central place in time series analysis has been reinforced by repeated successes against more sophisticated approaches. The manipulation with the importance of the last elements on the sequence and coupled with different smoothing strategies are developed, and these methods already demonstrated that predictors based on the evolutionary identification of near-optimal algebraic skeletons (which are neither linear nor stationary) are well applicable for non-linear and non-stationary sequences [6-8].

Many work have been done to enhance the prediction and simulation accuracy of ES. Some typical examples are as follows, the joint estimation of initial conditions and smoothing parameters can be achieved through maximum likelihood via box-constrained nonlinear optimization [9]. The ES method is extended to the case where the observations can have different significance in the smoothing process [10]. The interval-valued stock market time series are predicted by combining Holt smoothing method with a neural network [11]. A method for identifying outliers in ES by using innovations state space models has also been proposed [12].

DES is also known as Browns method. It is useful for series that exhibits a linear trend characteristic. Gardner summarized the history of DES [13]. A new jitter reduction scheme based on DES is proposed [14]. To resolve the conflict that exists between our desire for a good smoothing effect and desire to give additional weight to the recent change, we incorporate the grey generating operator into ES method in this paper. Grey generating operator is used to smooth the effect of random interference on the system. The smoothing constant is used to give additional weight to the recent change and to obtain minimal fitted error. Therefore, a novel grey ES method is put forward in Section 2. The advantages of grey ES method over the traditional model are illustrated by five real cases in Section 3. An empirical analysis of pig price in China is discussed in Section 4. Several conclusions are given in the last Section.

\section{Grey exponential smoothing}


As we know, for the original time series $X^{(0)}=\left\{x^{(0)}(1), x^{(0)}(2), \cdots, x^{(0)}(n)\right\}$, the single ES method follows the equation:

$$
\hat{x}^{(0)}(k+1)=\alpha x^{(0)}(k)+(1-\alpha) \hat{x}^{(0)}(k) .
$$

No formally correct procedure exists for choosing the smoothing coefficient, $0 \leq \alpha \leq 1$. Currently, the judgment of the statistician is used to obtain an ideal $\alpha$, which determines the level of response to recent changes and the smoothing effect. When $\alpha$ is near 0 , fewer responses to recent changes are observed and the smoothing effect is greater. When $\alpha$ is close to 1 , additional weight is given to the recent changes and the smoothing effect is lower [4,5]. This conflict deserves to be resolved. To solve this conflict, the following definition is given.

Definition 1 [15] For the original time series $X^{(0)}=\left\{x^{(0)}(1), x^{(0)}(2), \cdots, x^{(0)}(n)\right\}$, an $r$ order accumulated generating operator (AGO) sequence $X^{(r)}=\left\{x^{(r)}(1), x^{(r)}(2), \cdots, x^{(r)}(n)\right\}, r \in$ $R_{+}$can be generated by $r$-AGO as follows:

$$
x^{(r)}(k)=\sum_{i=1}^{k}\left(\begin{array}{c}
k-i+r-1 \\
k-i
\end{array}\right) x^{(0)}(i) ; k=1,2, \cdots, n .
$$

$\operatorname{Set}\left(\begin{array}{c}r-1 \\ 0\end{array}\right)=1,\left(\begin{array}{c}k-1 \\ k\end{array}\right)=0,\left(\begin{array}{c}k-i+r-1 \\ k-i\end{array}\right)=\frac{(r+k-i-1)(r+k-i-2) \cdots(r+1) r}{(k-i) !} . \quad r$-order inverse accumulated generating operator (IAGO) of $X^{(r)}$ is expressed as follows:

$$
X^{(-r)}={ }^{(\lceil r\rceil)} X^{(\lceil r\rceil-r)}=\left\{{ }^{(\lceil r\rceil)} x^{(\lceil r\rceil-r)}(1),{ }^{(\lceil r\rceil)} x^{(\lceil r\rceil-r)}(2), \cdots,{ }^{(\lceil r\rceil)} x^{(\lceil r\rceil-r)}(n)\right\}
$$

where $\lceil r\rceil=\min \{n \in Z \mid r \leq n\},{ }^{(\lceil r\rceil)} x^{(\lceil r\rceil-r)}(k)={ }^{(\lceil r-1\rceil)} x^{(\lceil r\rceil-r)}(k)-{ }^{(\lceil r-1\rceil)} x^{(\lceil r\rceil-r)}(k-1)$. The

IAGO is the inverse operation of AGO. In general, when $0<r<1, x^{(1)}(k)=\sum_{i=1}^{k} x^{(0)}(i)(k=$ $1,2, \cdots, n)$. The $r$-IAGO of $X^{(r)}$ is computed as follows:

$$
X^{(-r)}={ }^{(1)} X^{(1-r)}=\left\{{ }^{(1)} x^{(1-r)}(1),{ }^{(1)} x^{(1-r)}(2), \cdots,{ }^{(1)} x^{(1-r)}(n)\right\}
$$

where ${ }^{(1)} x^{(1-r)}(k)=x^{(1-r)}(k+1)-x^{(1-r)}(k)$.

AGO is widely used in grey models for its ability to smooth the randomness of original data [15-19]. By means of IAGO, the prediction value can be transformed back to the original se- 
quence. Through AGO, the disorderly data may be converted into regular form. For example, $X^{(0)}=\{1,2,1.5,3\}$, the 1-AGO sequence is $X^{(1)}=\{1,3,4.5,7.5\}$. The lines of these sequences are illustrated in Figs. 1 and 2 respectively. Comparing the two lines, it is clear that the trend of sequence $X^{(1)}$ in Fig.2 is more obvious than the sequence $X^{(0)}$ in Fig.1.

\section{[Insert Fig.1 about here]}

[Insert Fig.2 about here]

The following theorem is provided to discuss the mathematical property of AGO.

Theorem 1 Assume that $X^{(0)}=\left\{x^{(0)}(1), x^{(0)}(2), \cdots, x^{(0)}(n)\right\}$ is a nonnegative sequence. $X^{(r)}=\left\{x^{(r)}(1), x^{(r)}(2), \cdots, x^{(r)}(n)\right\}$ is the $r$-AGO sequence. If $r \geq 1, x^{(r)}(k)$ is the increasing function of $k(k=1,2, \cdots, n)$.

Proof For convenience, set $r=1, X^{(1)}=\left\{x^{(1)}(1), x^{(1)}(2), \cdots, x^{(1)}(n)\right\}=\left\{x^{(0)}(1), x^{(0)}(1)+\right.$ $\left.x^{(0)}(2), \cdots, x^{(0)}(1)+x^{(0)}(2)+\cdots+x^{(0)}(n)\right\}$. Obviously, $x^{(1)}(n)>x^{(1)}(n-1)>\cdots>x^{(1)}(2)>$ $x^{(1)}(1)$. If $r=2, X^{(2)}=\left\{x^{(2)}(1), x^{(2)}(2), \cdots, x^{(2)}(n)\right\}=\left\{x^{(0)}(1), 2 x^{(0)}(1)+x^{(0)}(2), \cdots, n x^{(0)}(1)+\right.$ $\left.(n-1) x^{(0)}(2)+\cdots+x^{(0)}(n)\right\}$. Obviously, $x^{(2)}(n)>x^{(2)}(n-1)>\cdots>x^{(2)}(2)>x^{(2)}(1)$. Actually, $\sum_{i=1}^{k}\left(\begin{array}{c}k-i+r-1 \\ k-i\end{array}\right) x^{(0)}(i)-\sum_{i=1}^{k-1}\left(\begin{array}{c}k-2-i+r \\ k-1-i\end{array}\right) x^{(0)}(i)=x^{(0)}(k)+\sum_{i=1}^{k-1}\left(\begin{array}{c}k-2-i+r \\ k-i\end{array}\right) x^{(0)}(i)>0$, i.e, $x^{(r)}(k)>x^{(r)}(k-1)$. Thus, $x^{(r)}(k)$ is the increasing function of $k(k=1,2, \cdots, n)$.

Actually, If $0<r<1, x^{(r)}(k)$ may be the increasing function of $k(k=1,2, \cdots, n)$. For example, an irregular sequence $X^{(0)}=\{6,4,7,5,6,4,10,9,11,10\}$, the 0.6 -AGO sequence of $X^{(0)}$ is $X^{(0.6)}=\{6,7.6,12.28,13.61,16.27,16.48,23.29,26.80,32.02,35.18\}$. We can see that $x^{(0.6)}(k)$ is the increasing function of $k(k=1,2, \cdots, 10)$. By using AGO, the disorderly sequence may be converted into an approximately increased sequence. An irregular and increased sequence can be predicted by using Holt's linear method (double exponential smoothing). Then we give the following definition:

Definition 2 For the original time series $X^{(0)}=\left\{x^{(0)}(1), x^{(0)}(2), \cdots, x^{(0)}(n)\right\}, r$-AGO is 
given in Definition 1. Grey double exponential smoothing (GDES) follows the equations

$$
\begin{gathered}
S^{\prime}(k)=\alpha x^{(r)}(k)+(1-\alpha) S^{\prime}(k-1) \\
S^{\prime \prime}(k)=\alpha S^{\prime}(k)+(1-\alpha) S^{\prime \prime}(k-1) \\
b_{k}=\frac{\alpha}{1-\alpha}\left(S^{\prime}(k)-S^{\prime \prime}(k)\right) \\
a_{k}=2 S^{\prime}(k)-S^{\prime \prime}(k)
\end{gathered}
$$

where $S^{\prime}(k)$ and $S^{\prime \prime}(k)$ are the single and double exponential smoothing values for time $k$ respectively. The forecasting form is $x^{(r)}(k)=a_{k}+k b_{k}$.

If $r=0$, GDES is the traditional double exponential smoothing. The process of calculating GDES can be summarized as follows:

Step 1: Set the order number $r$ and obtain the $r$-AGO sequence of $X^{(0)}$ according to Definition 1

Step 2: Calculate the parameters $\left(a_{k}\right.$ and $\left.b_{k}\right)$ by using Definition 2;

Step 3: Compute the predictive value using the following equation $\hat{x}^{(r)}(k+m)=a_{k}+m b_{k}$, where $m$ is the out-of-sample size;

Step 4: Transform the prediction value back to the original sequence by means of IAGO.

The following theorem is provided to discuss the mathematical property of GDES.

Theorem 2 Assume that $X^{(0)}=\left\{x^{(0)}(1), x^{(0)}(2), \cdots, x^{(0)}(n)\right\}$ is a time series. $X^{(r)}=$ $\left\{x^{(r)}(1), x^{(r)}(2), \cdots, x^{(r)}(n)\right\}$ is the $r$-AGO sequence and $r$ is not an integer. Construct GDES according to the above steps. $m$ is the out-of-sample size, then $\hat{x}^{(r)}(k)$ is the weighted linear function of one variable $k(k=1,2, \cdots, n, n+1, \cdots, n+m)$, and the monotonicity of the predictive value $\hat{x}^{(0)}(k)(k=n, n+1, \cdots, n+m)$ is uncertain.

Proof For convenience, set $r=\frac{p}{q}, \frac{p}{q}$-AGO can be expressed as follows: 


$$
\left[x^{\left(\frac{p}{q}\right)}(1), x^{\left(\frac{p}{q}\right)}(2), \cdots, x^{\left(\frac{p}{q}\right)}(n)\right]=\left[x^{(0)}(1), x^{(0)}(2), \cdots, x^{(0)}(n)\right]\left[\begin{array}{ccccc}
1 & C_{\frac{p}{q}}^{1} & \cdots & C_{\frac{p}{q}+n-3}^{n-2} & C_{\frac{p}{q}+n-2}^{n-1} \\
0 & 1 & \cdots & C_{\frac{p}{q}+n-4}^{n-3} & C_{\frac{p}{q}+n-3}^{n-2} \\
\vdots & \vdots & \vdots & \vdots & \vdots \\
0 & 0 & \cdots & 1 & C_{\frac{p}{q}}^{1} \\
0 & 0 & \cdots & 0 &
\end{array}\right]
$$

Therefore, $\frac{p}{q}$-IAGO can be expressed as follows:

$$
\begin{gathered}
{\left[\hat{x}^{(0)}(1), \hat{x}^{(0)}(2), \cdots, \hat{x}^{(0)}(n)\right]=\left[\hat{x}^{\left(\frac{p}{q}\right)}(1), \hat{x}^{\left(\frac{p}{q}\right)}(2), \cdots, \hat{x}^{\left(\frac{p}{q}\right)}(n)\right]\left[\begin{array}{cccc}
1 & -C_{\frac{p}{q}}^{1} & \cdots & (-1)^{n-1} C_{\frac{p}{q}}^{n-1} \\
0 & 1 & \cdots & (-1)^{n-2} C_{\frac{p}{q}}^{n-2} \\
\vdots & \vdots & \vdots & \vdots \\
0 & 0 & \cdots & -C_{\frac{p}{q}}^{1} \\
0 & 0 & \cdots & 1
\end{array}\right]} \\
=\left[a_{1}+b_{1}, a_{2}+b_{2}, \cdots, a_{n}+b_{n}\right]\left[\begin{array}{cccc}
1 & -C_{\frac{p}{q}}^{1} & \cdots & (-1)^{n-1} C_{\frac{p}{q}}^{n-1} \\
0 & 1 & \cdots & (-1)^{n-2} C_{\frac{p}{q}}^{n}-2 \\
\vdots & \vdots & \vdots & \vdots \\
0 & 0 & \cdots & -C_{\frac{p}{q}}^{1} \\
0 & 0 & \cdots & 1
\end{array}\right]
\end{gathered}
$$

The predictive value of $\frac{p}{q}$-IAGO can be expressed as follows:

$$
\begin{gathered}
{\left[\hat{x}^{(0)}(1), \hat{x}^{(0)}(2), \cdots, \hat{x}^{(0)}(n), \cdots, \hat{x}^{(0)}(n+m)\right]} \\
=\left[a_{1}+b_{1}, a_{2}+b_{2}, \cdots, a_{n}+b_{n}, a_{n}+2 b_{n}, \cdots, a_{n}+m b_{n}\right]\left[\begin{array}{cccc}
1 & -C_{\frac{p}{q}}^{1} & \cdots & (-1)^{n+m-1} C_{\frac{p}{q}}^{n+m-1} \\
0 & 1 & \cdots & (-1)^{n+m-2} C_{\frac{p}{q}}^{n+m-2} \\
\vdots & \vdots & \vdots & \vdots \\
0 & 0 & \cdots & -C_{\frac{p}{q}}^{1} \\
0 & 0 & \cdots & 1
\end{array}\right]
\end{gathered}
$$

From the above equation we conclude that the predictive sequence $\hat{x}^{(0)}(k)(k=n, n+1, \cdots, n+$ $m$ ) is a weighted linear model and that these weights are changeable. Therefore, the monotonicity of the predictive value $\hat{x}^{(0)}(k)(k=n, n+1, \cdots, n+m)$ is uncertain.

In this paper, the predictive values are calculated by using different $\alpha$ 's. The $\alpha$ that produces a small mean square error for the fitted values and shows an expected future growth is chosen. We assumed that $S^{\prime}(0)$ and $S^{\prime \prime}(0)$ are equal to the initial historical values. 


\section{Comparison of traditional exponential smoothing and GDES}

The effectiveness of the GDES model is evaluated via five real cases in this section. Mean

absolute percentage error (MAPE $\left.=100 \% \frac{1}{n} \sum_{k=1}^{n}\left|\frac{x^{(0)}(k)-\hat{x}^{(0)}(k)}{x^{(0)}(k)}\right|\right)$ compares the actual values with the forecasted values to evaluate the precision.

\section{Case 1: The example for the series N8 from the M3 forecasting competition}

Take the series N8 from the M3 forecasting competition as example. The last 6 observations is not be used to calculate the forecasts but to evaluate their accuracy. The forecasting results and the growth values are shown in Table 1.

Table 1 The results of different models

\begin{tabular}{cccccccc}
\hline Year & Actual value & Holt & growth value & $\begin{array}{c}\text { DES } \\
\alpha=0.51\end{array}$ & growth value & $\begin{array}{c}\text { GDES } \\
\alpha=0.51\end{array}$ & growth value \\
\hline 1989 & 8809.8 & 9809.8 & & 10994.5 & & 7184.6 & \\
1990 & 8953.5 & 12517.9 & 2708.1 & 12421.3 & 1426.8 & 7105.2 & -79.4 \\
1991 & 7991.01 & 15225.9 & 2708.1 & 13848.2 & 1426.8 & 7170.0 & 65.8 \\
1992 & 7294.08 & 17933.0 & 2708.1 & 15275.0 & 1426.8 & 7270.5 & 99.5 \\
1993 & 7163.37 & 20642.0 & 2708.1 & 16701.8 & 1426.8 & 7378.5 & 108.0 \\
1994 & 7478.31 & 23350.1 & 2708.1 & 18128.6 & 1426.8 & 7486.0 & 108.5 \\
MAPE & & 114.7 & & 87.0 & & 8.8 & \\
\hline
\end{tabular}

It is noted that the growth values of forecasting results by GDES are changeable. But the growth values of forecasting results by DES (Holt) are constant. Therefore, GDES can obtain the smallest MAPE and reflect the real system with different growth situations. This is a major innovation of GDES.

\section{Case 2: The example for the incidence of Hepatitis B [18]}

This example is from paper [18]. The in-sample data and out-of-sample data are the same as the paper [18]. The actual value and the errors of five models are shown in Table 2. On the one hand, DES yields the lowest MAPE among the five in-sample models, but yields the higher MAPE 
among the out-of-sample models. On the other hand, GDES yields the lower MAPE among the five in-sample models, but the lowest MAPE among the out-of-sample models. It implies that GDES can mitigate the drawbacks of DES and can produce better forecasting results by capturing the characteristics of recent data.

Table 2 The fitting values and MAPE of different models

\begin{tabular}{ccccccc}
\hline Month & Actual value & GM(1,1) & Improved model ${ }^{[18]}$ & Holt-Winters ${ }^{[18]}$ & GDES & DES \\
\hline Jan & 162818 & & & & & \\
Feb & 214523 & 200801 & 214523 & 214523 & 228120 & 224864 \\
Mar & 201184 & 193817 & 188870 & 201184 & 221737 & 215062 \\
Apr & 155942 & 187077 & 178600 & 155942 & 161127 & 154207 \\
May & 183216 & 180571 & 173594 & 183216 & 183707 & 177824 \\
Jun & 165935 & 174291 & 171250 & 162818 & 167469 & 162806 \\
Jul & 175836 & 168229 & 170520 & 216749 & 177048 & 173411 \\
Aug & 170885 & 162379 & 170885 & 174242 & 172907 & 170040 \\
MAPE & & 6.5 & 4.6 & 3.4 & 3.2 & 2.8 \\
\hline Sep & 162818 & 156731 & 172054 & 126074 & 173099 & 168790 \\
Oct & 133160 & 151281 & 173849 & 152772 & 162379 & 167541 \\
Nov & 190779 & 146019 & 176156 & 131799 & 173792 & 166291 \\
Dec & 178907 & 140941 & 178897 & 182929 & 174323 & 165041 \\
MAPE & & 15.5 & 11.0 & 17.6 & 9.9 & 12.5 \\
\hline
\end{tabular}

\section{Case 3: The numeric example [20]}

Li used this numeric example to discuss the conflict between our desire for a good smoothing effect and desire to give additional weight to the recent change [20]. Despite explaining the existence of this conflict, Li did not suggest a method for solving such a conflict. To compare the smoothing effect of GDES, the smoothing constant of $\operatorname{Li}(\alpha=0.8)$ is used. The results of three models are shown in Table 3. As shown in Table 3, the MAPE of GDES is the smallest among the three 
models. This shows that GDES can mitigate the drawbacks of the traditional single exponential smoothing and double exponential smoothing method.

Table 3 The results of different models

\begin{tabular}{ccccc} 
Time & Actual value & Traditional single $\operatorname{ES}^{[20]}(\alpha=0.8)$ & $\operatorname{GDES}(\alpha=0.8)$ & DES \\
\hline 1 & 6 & & 3.9 & 2.8 \\
2 & 4 & 6 & 9.1 & 8.2 \\
3 & 7 & 6.4 & 5.0 & 4.5 \\
4 & 5 & 5.3 & 6.6 & 6.3 \\
5 & 6 & 5.9 & 3.2 & 3.0 \\
6 & 4 & 4.4 & 13.3 & 13.1 \\
7 & 10 & 8.9 & 10.1 & 9.9 \\
8 & 9 & 9.0 & 12.5 & 12.4 \\
9 & 11 & 10.6 & 10.1 & 10.0 \\
10 & 10 & 25.8 & 12.2 & 14.1 \\
\hline
\end{tabular}

\section{Case 4: The gas content forecasting example [21]}

He et al. used the linear double exponential smooth method to predict gas content in vertical depth with an interval of $50 \mathrm{~m}$ in a mining area [21]. Their results showed the linear double exponential smooth method with a smooth constant $(\alpha=0.8)$ achieved a high prediction accuracy. To compare the smoothing effect of GDES, the smoothing constant of Reference [21] $(\alpha=0.8)$ is used. The results of the four models are shown in Table 4. As shown in Table 4, GDES $(\alpha=0.4)$ achieves the lowest MAPE among the four models, which implies that GDES can obtain better results than the other models. 
Table 4 The results of different models

\begin{tabular}{cccccc} 
Order & Actual value & $\operatorname{DES}^{[21]}(\alpha=0.8)$ & $\operatorname{GDES}(\alpha=0.8)$ & $\operatorname{GDES}(\alpha=0.4)$ & $\operatorname{GM}(1,1)$ \\
\hline 1 & 5.35 & 5.82 & 6.13 & 5.62 & 4.21 \\
2 & 5.82 & 6.10 & 7.36 & 6.58 & 5.18 \\
3 & 6.68 & 7.33 & 4.95 & 5.98 & 6.37 \\
4 & 5.41 & 4.93 & 9.29 & 7.77 & 7.83 \\
5 & 7.99 & 9.27 & 9.16 & 8.74 & 9.63 \\
6 & 8.32 & 9.15 & 13.81 & 11.63 & 11.84 \\
7 & 11.56 & 13.80 & 20.62 & 16.70 & 14.56 \\
8 & 16.6 & 16.5 & 11.8 & 3.1 & 16.3 \\
\hline
\end{tabular}

Case 5: The examples for the series N1, N2, N3, N4, and N5 from the M3 forecasting competition

The series N1, N2, N3, N4 and N5 are from the M3 forecasting competition. The last 6 observations are not be used to calculate the forecasts but to evaluate their accuracy. The forecasting results of the series N1, N2, N3, N4 and N5 are listed in Table 5, Table 6, Table 7 Table 8 and Table 9, respectively. The lines of these sequences are illustrated in Figs.3-7 respectively.

Table 5 The results of different models

\begin{tabular}{|c|c|c|c|c|c|}
\hline Year & Actual value & $\operatorname{GM}(1,1)$ & ARIMA & $\operatorname{DES}(\alpha=0.98)$ & $\operatorname{GDES}(\alpha=0.98)$ \\
\hline 1989 & 5379.75 & 5564.0 & 5553.26 & 6037.4 & 6035.5 \\
\hline 1990 & 6158.68 & 6248.3 & 6246.45 & 6587.5 & 6582.5 \\
\hline 1991 & 6876.58 & 7016.7 & 7026.18 & 7137.7 & 7128.9 \\
\hline 1992 & 7851.91 & 7879.6 & 7903.23 & 7687.9 & 7674.8 \\
\hline 1993 & 8407.84 & 8848.7 & 8889.77 & 8238.1 & 8220.2 \\
\hline 1994 & 9156.01 & 9936.9 & 9999.45 & 8788.3 & 8765.2 \\
\hline MAPE & & 3.5 & 3.7 & 5.2 & 5.2 \\
\hline
\end{tabular}




$$
\begin{aligned}
& \text { [Insert Fig.4 about here] } \\
& \text { [Insert Fig.5 about here] } \\
& \text { [Insert Fig.6 about here] } \\
& \text { [Insert Fig. } 7 \text { about here] }
\end{aligned}
$$

Take the series N1 from the M3 forecasting competition as an example. According to Table 5 and Fig.3, $\operatorname{GM}(1,1)$ has the smallest MAPE among the four models. Therefore, the series N1 shows an evident exponential trend. The error rate of GDES model is very close to DES model. Thus, the series N1 is unsuitable for GDES.

Table 6 The results of different models

\begin{tabular}{cccccc} 
Year & Actual value & ARIMA & DES $(\alpha=0.1)$ & GDES $(\alpha=0.1)$ & Auto-ANN \\
\hline 1989 & 4793.2 & 4287.75 & 4949.6 & 4908.7 & 4391.67 \\
1990 & 5602 & 4346.3 & 5048.1 & 4958.3 & 4513.11 \\
1991 & 5065 & 4405.64 & 5146.6 & 5014.5 & 4604.32 \\
1992 & 5056 & 4465.79 & 5245.0 & 5075.8 & 4672.83 \\
1993 & 5067.2 & 4526.76 & 5343.5 & 5140.9 & 4724.29 \\
1994 & 5209.6 & 4588.57 & 5442.0 & 5209.1 & 4762.95 \\
MAPE & & 13.4 & 4.7 & 2.7 & 10.0 \\
\hline & & & & & \\
\hline & & & & & \\
Year & Actual value & ARIMA & DES $(\alpha=0.8)$ & GDES $(\alpha=0.8)$ & Auto-ANN \\
\hline 1989 & 3070.2 & 3558.91 & 3790.44 & 3464.11 & 3230.66 \\
1990 & 3601.6 & 3592.94 & 3933.13 & 3437.34 & 3232.3 \\
1991 & 3407.4 & 3627.3 & 4075.81 & 3432.16 & 3231.2 \\
1992 & 3500.6 & 3661.98 & 4218.49 & 3434.63 & 3230.75 \\
1993 & 3437.8 & 3697 & 4361.17 & 3441.41 & 3231.25 \\
1994 & 3007 & 3732.35 & 4503.85 & 3451.22 & 3231.24 \\
MAPE & & 9.8 & 24.9 & 5.8 & 7.0 \\
\hline
\end{tabular}


Table 8 The results of different models

\begin{tabular}{cccccc} 
Year & Actual value & ARIMA & DES $(\alpha=0.52)$ & GDES $(\alpha=0.52)$ & Auto-ANN \\
\hline 1989 & 4656 & 4878.4 & 4961.9 & 4725.90 & 4132.45 \\
1990 & 5228.52 & 5137.57 & 5235.05 & 4865.27 & 4079.99 \\
1991 & 5656.72 & 5410.51 & 5508.16 & 5021.65 & 4072.9 \\
1992 & 5077.02 & 5697.95 & 5781.27 & 5181.69 & 4071.91 \\
1993 & 5403.4 & 6000.66 & 6054.37 & 5341.91 & 4071.77 \\
1994 & 5009.52 & 6319.45 & 6327.48 & 5501.14 & 4071.75 \\
MAPE & & 10.1 & 10.3 & 5.4 & 20.7 \\
\hline
\end{tabular}

Table 9 The results of different models

\begin{tabular}{ccccc} 
Year & Actual value & naïve2 & $\operatorname{DES}(\alpha=0.13)$ & GDES $(\alpha=0.13)$ \\
\hline 1989 & 5250.9 & 5488.8 & 5238.3 & 4782.5 \\
1990 & 4899.2 & 5488.8 & 5228.9 & 4598.2 \\
1991 & 4317.9 & 5488.8 & 5219.5 & 4511.5 \\
1992 & 4007.9 & 5488.8 & 5210.2 & 4463.2 \\
1993 & 4323.4 & 5488.8 & 5200.8 & 4435.3 \\
1994 & 4819.4 & 5488.8 & 5191.4 & 4419.9 \\
MAPE & & 20.2 & 14.3 & 6.9 \\
\hline
\end{tabular}

From the results of the series N2, N3, N4 and N5. It is very clear that the accuracies of GDES models are better than the conventional models'. The results validate the effectiveness of GDES.

Compare Table 6-9 with Table 5, we conclude that the series N2, N3, N4 and N5 are suitable for GDES and the series N1 is unsuitable for GDES. Compare Fig.4-7 with Fig.3, the area of GDES applicability is the linear trend with some fluctuating data.

\section{Pig price forecasting in China}

Pork is an important element in the Chinese food culture. On average day in China, approximately 1703975 pigs are consumed. According to a report by the United States Department of Agriculture, Chinese consumed 51 million metric tons of pork in 2006, roughly half of the total pig 
consumption in the world. Although pork accounts for just 3 percent of the products used to measure CPI, huge fluctuations in pork price can significantly change CPI. Therefore, CPI has often been referred to as "the China Pork Index". Over the recent years, pig price have demonstrated abnormal fluctuations that have raised widespread concern in China. Therefore, the accurate forecasting of pig price is very important.

Given the limited historical data is available, traditional regression analysis is unsuitable for forecasting pig price. Instead, the grey forecasting model and ES method can be used to predict pig price [22]. However, the grey forecasting model is an exponential curve and DES method is a linear model. These two methods are not suitable for forecasting pig price, because the pig price usually represents an abnormal surge. Therefore, given the non-deterministic and limited-samples condition, we use GDES to predict the pig price.

The data consist of yearly pig price in China from 2004 to 2010. These real data were collected from http://www.caaa.cn. The original time series is shown as follows:

$$
X^{(0)}=\{8.70,8.05,7.21,11.84,14.87,11.25,11.50,16.88,15.20,14.56\}
$$

The 0.75-AGO sequence is expressed as follows:

$$
X^{(0.1)}=\{8.70,8.92,8.49,13.34,17.02,14.12,14.49,20.08,19.12,18.78\} .
$$

We calculate $a_{k}=2 S^{\prime}(k)-S^{\prime \prime}(k), b_{k}=\frac{\alpha}{1-\alpha}\left(S^{\prime}(k)-S^{\prime \prime}(k)\right)$, where

$$
S^{\prime}(k)=0.75 x^{(r)}(k)+(1-0.75) S^{\prime}(k-1), S^{\prime \prime}(k)=0.75 S^{\prime}(k)+(1-0.75) S^{\prime \prime}(k-1) .
$$

Therefore, we obtain $a_{n}=18.88, b_{n}=0.13$.

$$
\begin{aligned}
\hat{X}^{(0.1)} & =\{8.70,9.03,8.35,15.66,20.33,14.41,14.43,22.84,20.38,19.01,19.14,19.27,19.39\} . \\
\hat{X}^{(0.1)(0.9)} & =\{8.70,16.86,23.91,38.08,56.03,67.14,78.34,97.84,113.91,128.36,142.84,157.29,171.71\} .
\end{aligned}
$$

Then

$$
\hat{X}^{(0)}=\{8.70,8.16,7.05,14.17,17.95,11.11,11.20,19.50,16.07,14.45,14.48,14.45,14.42,15.04\} .
$$


Table 10 The results of different models

\begin{tabular}{cccc} 
Year & Actual value & GDES $(\alpha=0.75)$ & GM $(1,1)$ \\
\hline 2004 & 8.70 & & \\
2005 & 8.05 & 8.16 & 9.14 \\
2006 & 7.21 & 7.05 & 9.82 \\
2007 & 11.84 & 14.17 & 10.56 \\
2008 & 14.87 & 17.95 & 11.35 \\
2009 & 11.25 & 11.11 & 12.19 \\
2010 & 11.50 & 11.20 & 13.11 \\
2011 & 16.88 & 19.50 & 14.08 \\
2012 & 15.20 & 16.07 & 15.14 \\
2013 & 14.56 & 14.45 & 16.27 \\
MAPE & & 6.98 & 14.72 \\
\hline
\end{tabular}

The simulative values and their errors are shown in Table 10. According to Table 10, GDES has a strong performance in forecasting of pig price in the context of short term or small sample, because the MAPE is more smaller than $\operatorname{GM}(1,1)$, which is a remarkable advantage of GDES. Thus we use GDES to predict short-term pig price. The results are listed in Table 11.

Table 11 The forecasting results of Pig price in China

\begin{tabular}{cc} 
Year & $\operatorname{GDES}(\alpha=0.75)$ value \\
\hline 2014 & 14.48 \\
2015 & 14.45 \\
2016 & 14.42 \\
2017 & 15.04 \\
\hline
\end{tabular}

The results of Table 11 shows that the pig price will demonstrate small fluctuations, which closely reflect the actual situation in the country. Judging by the current state of the domestic pig farming industry, the pig prices in China will remain stabilized for a specific period, because China possesses a national pork reserve. Unless a new disease breakout leads to a steep cull of pig stocks, 
the pork prices in China will not experience huge fluctuations. If the pig price in China has been pushed up. According to the Ministry of Commerce, there are 200000 tonnes of pork in reserves in 2011, which will be released into the market as and when needed. If the pig price continues to decrease, driving the food CPI below zero. The government will launch a round of domestic frozen pork purchases to prevent any further drops in the pork price, as well as to protect the interest of farmers.

\section{Discussion and Conclusion}

$r$ can change the smoothing effect of GDES as well as determine the linear trend of $r$-AGO.

The smoothing constant can provide additional weight to the recent changes. Although favorable results have been obtained from the six cases, $r$ may not be optimal. The performance of the GDES still could be improved by further advancement of algorithms used to determine $r$. More computational experiments are needed to make conclusions on the performance of different orders. How to simply obtain the optimal $r$ that can produce a minimum error is a problem that deserves to research in the future. It is quite probable that the forecasting accuracy of the GDES can be improved by introduction of external-internal-mixed smoothing.

The government has begun to stockpile frozen pork to stabilize pig prices and to protect pig breeders from losses and frustration when the price of pig has fallen. The government will release part of the central government's frozen pork into the market to cap the increasing prices when the pig price has fallen. Therefore, the pig price will demonstrate a stabilized trend in the short term, and the results of GDES are proven accurate. GDES is eventually validated as a better forecasting model for pig prices and may be used to predict in other real cases. This model can also be extended to triple exponential smoothing in the future.

\section{Acknowledgements}


The relevant researches done are supported by a Marie Curie International Incoming Fellowship within the 7th European Community Framework Programme (No.FP7-PIIF-GA-2013-629051) and National Natural Science Foundation of China (No.71401051).

\section{References}

[1] Holt, C. C. Forecasting seasonals and trends by exponentially weighted moving averages. International Journal of Forecasting 2004,20:5-13.

[2] James W. Taylor. Density forecasting of intraday call center arrivals using models based on exponential smoothing. Management Science 2012,58(3):534-549.

[3] G. Sudheer, A. Suseelatha. Litteral. Short term load forecasting using wavelet transform combined with Holt-Winters and weighted nearest neighbor models. Electrical Power and Energy Systems 2015,64:340-346.

[4] Ward Romeijnders, Ruud Teunter, Willem van Jaarsveld. A two-step method for forecasting spare parts demand using information on component repairs. European Journal of Operational Research 2012, 220(2): 386-393.

[5] Guiling Li, Zhihua Cai, Xiaojun Kang, et al. ESPSA: A prediction-based algorithm for streaming time series segmentation. Expert Systems with Applications 41(2014):6098-6105.

[6] Minvydas Ragulskis, Kristina Lukoseviciute, Zenonas Navickas, et al. Short-term time series forecasting based on the identification of skeleton algebraic sequences. Neurocomputing 2011, 74:1735-1747.

[7] Rita Palivonaite, Minvydas Ragulskis. Short-term time series algebraic forecasting with internal smoothing. Neurocomputing 2014, 127:161-171. 
[8] Rita Palivonaite, Kristina Lukoseviciute,Minvydas Ragulskis. Short-term time series algebraic forecasting with mixed smoothing. Neurocomputing 2015, doi:10.1016/j.neucom.2015.07.018.

[9] E.Vercher, A.Corberan-Vallet, J.V.Segura, et al. Initial conditions estimation for improving forecast accuracy in exponential smoothing. Top 2012,20:517-533.

[10] Ronald R. Yager. Exponential smoothing with credibility weighted observations. Information Sciences 2013(252): 96-105.

[11] Andre Luis Santiago Maia, Francisco de A.T. de Carvalho. Holt's exponential smoothing and neural network models for forecasting interval-valued time series. International Journal of Forecasting 2011(27): 740-759.

[12] Anne B. Koehler, Ralph D. Snyder, J. Keith Ord, et al. A study of outliers in the exponential smoothing approach to forecasting. International Journal of Forecasting 2012(28): 477-484.

[13] Everette S. Gardner Jr. Exponential smoothing: The state of the art-Part II. International Journal of Forecasting 22 (2006):637-666.

[14] Min Gyo Chung, Sang-Kyun Kim. Efficient jitter compensation using double exponential smoothing. Information Sciences 227 (2013):83-89.

[15] Lifeng Wu, Sifeng Liu, Ligen Yao, et al. Grey system model with the fractional order accumulation. Communications in Nonlinear Science and Numerical Simulation 2013,18(7):17751785.

[16] Li D.C, Chang C.J, Chen C.C, et al. Forecasting short-term electricity consumption using the adaptive grey-based approach-An Asian case. Omega 2012,40(6): 767-773. 
[17] Ling-Ming Kung, Shang-Wu Yu. Prediction of index futures returns and the analysis of financial spilloversA comparison between GARCH and the grey theorem. European Journal of Operational Research 2008,186(3): 1184-1200.

[18] Liping Zhang, Yanling Zheng, Kai Wang, et al. An optimized Nash nonlinear grey Bernoulli model based on particle swarm optimization and its application in prediction for the incidence of Hepatitis B in Xinjiang, China. Computers in Biology and Medicine 2014,49(1):67-73.

[19] Ze Zhao, Jianzhou Wang, JingZhao, et al. Using a grey model optimized by differential evolution algorithm to forecast the per capita annual net income of rural households in China. Omega 2012,40(5):525-532.

[20] Li Baoren. Theory and Application of Economic Forecasting. Economy \& Management Publishing House, Beijing 2005.

[21] He Jun, Liu Linbing, Tang Yiju.Gas content prediction based on linear double exponential smooth method. Coal Science and Technology 2014,42(12):48-50.

[22] Hao Miao, Fu Xinhong, Chen Rong. Application of grey system theory in pig price prediction. Chinese Agricultural Science Bulletin 2014,30(14):310-314. 